\title{
Effects of Temperature on the Free Carrier Traps of Shockley Read Hall Recombination Mechanisms for Gallium Sulfide (GaS) Semiconductor
}

\author{
G.E.Gebramichael \\ Department of Physics, CNCS, Wolkite University, P.O.Box 07, Wolkite, Ethiopia
}

Received December 5, 2019; Revised January 29, 2020; Accepted February 7, 2020

Copyright $\odot 2020$ by authors, all rights reserved. Authors agree that this article remains permanently open access under the terms of the Creative Commons Attribution License 4.0 International License

\begin{abstract}
In this paper, we have studied the effects of temperature on the free carrier trap of Gallium Sulfide $(\mathrm{GaS})$ in Shockley read hall recombination mechanism. We have seen dependencies on the energy level in the trap of electrons and holes in their respective bands and classify these energy levels into five regions based on the interactions of the localized states with the conduction or valence bands in the high-temperature region. Gallium sulfide compound semiconductor has material impurities that introduce some intermediate energy levels in the forbidden gap. These levels act as recombination centers (or traps, from which the process is otherwise depicted as trap assisted recombination) and an intermediate step is introduced in the recombination process. Another is described as a hole that ascends to the intermediate level (equivalently described as a hole capture of the trap), recombining with an electron. It has also investigated that, at high temperatures, it shows only the electron trap for a wider range of localized trap energies and it shows a small region of localized trap energies for low temperatures. It also shows similar variations for trap density. Additionally, we also analyse the effects of injection levels on traps of free carriers. The variation of both excess carriers in the conduction and valence bands and excess electrons on trap level is the same with illumination at low injection level.
\end{abstract}

Keywords Shockley Read Hall, Gallium Sulfide, Temperature, Carriers Trap, Injection Level

\section{Introduction}

Gallium Sulfide $(G a S)$ is one of the most important wide band gap III-VI semiconductors $\left(E_{g}=3.05 \mathrm{eV}\right)$ with layered structures. GaS usually crystallizes into double layers of nonmetal atoms, each consisting of [S-Ga-Ga-S] sheets and stacking together by the non-bonding interaction through the $S$ atoms along the c-axis. There are two layers in a single GaS unit cell, in which the bonding between two adjacent layers is the Vander Waal interaction and the bonding within a layer is predominantly covalent. The strong intra layer bonding and the weak inter-layer van der Waals interaction give rise to highly anisotropic structural, electrical, optical, and mechanical properties, which have made GaS attractive in photoelectric devices, electrical sensors, and nonlinear optical applications [1].

GaS belongs to a family of semiconductor crystals along with $\mathrm{GaSe}$ and InSe, which crystallize in a layered structure. In $G a S$, inter-layer interactions are dominated by van der Waals forces, while intra layer-bonding forces are primarily ionic covalent. Due to the weak interlayer interaction, $\mathrm{GaS}$ can easily be cleaved along with these layers [2].

A direct band gap of $\mathrm{GaS}$ exists at approximately $0.45 \mathrm{eV}$ higher in energy. Thus, GaS is considered to be a promising material for near-blue light-emitting devices. It exhibits both electroluminescence and PL in the green-blue region [3, 4]. $\mathrm{PL}$ of both undoped and $\mathrm{Zn}$ doped $\mathrm{GaS}$ near the fundamental band edge has been studied by various authors $[5,6]$. Near band edge luminescence attributed to recombination of shallow donors with distant acceptors has been proposed [7].

Over the last decades, organic electronics have become a field of material science advancing rapidly into the commercial domain. Knowledge of the physical processes governing the operation of organic devices is essential for optimizing their performance [8]. Recombination of electrons and holes in organic semiconductors is an important process; it determines the efficiency of organic light-emitting diodes (OLEDs) and is an unwanted loss process in organic solar cells [9].

Another important recombination process that dominates the recombination in indirect semiconductors as silicon and germanium is trap-assisted recombination. This is a two-step process where a trap state, originating from imperfections or impurities in the crystal structure, creating energy levels inside 
the forbidden energy band gap, captures a charge carrier that subsequently recombines with a mobile carrier of the opposite sign because of their Coulombic interaction. Because of conservation of momentum this process cannot occur without the release of a phonon slowing the recombination process down. Therefore, in most cases the trap sites act as recombination centers for non-radiative recombination. Trap assisted recombination was first described by Shockley, Read, and Hall (SRH) in 1952 [10].

The Shockley-Read-Hall (SRH) model was introduced to describe the statistics of recombination and generation of holes and electrons in semiconductors occurring through the mechanism of trapping. The transfer of electrons from the valence band to the conduction band is referred to as the generation of electron-hole pairs (or pair-generation process), since not only is a free electron created in the conduction band, but also a hole in the valence band which can contribute to the charge current. The inverse process is termed recombination of electron-hole pairs. The band gap between the upper edge of the valence band and the lower edge of the conduction band is very large in semiconductors, which means that a big amount of energy is needed for a direct band-to-band generation event. The presence of trap levels within the forbidden band caused by crystal impurities facilitates this process, since the jump can be split into two parts, each of them "cheaper" in terms of energy. The basic mechanisms are illustrated in Figure 1: (a) hole emission (an electron jumps from the valence band to the trapped level), (b) hole capture (an electron moves from an occupied trap to the valence band, and a hole disappears), (c) electron emission (an electron jumps from the trapped level to the conduction band), (d) electron capture (an electron moves from the conduction band to an unoccupied trap) [12].

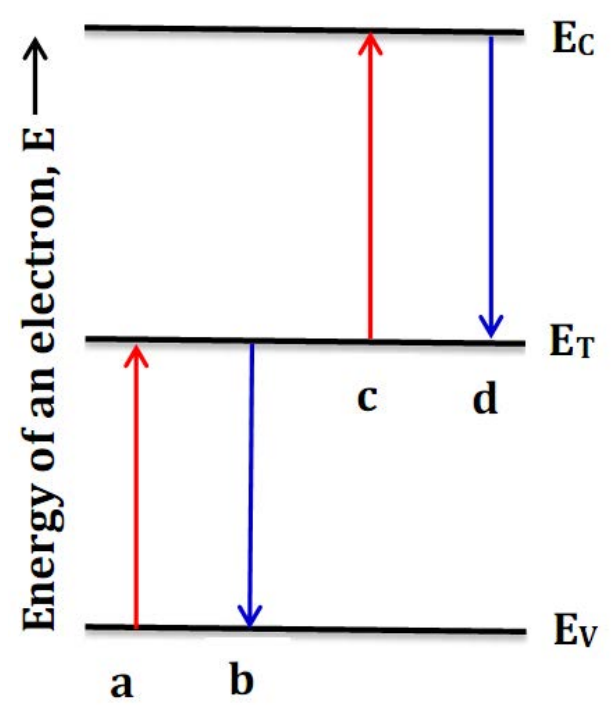

Figure 1. The four basic processes of electron-hole recombination.

In this article, we report the temperature dependence of free carriers in the Shockley read hall recombination mechanism by considering trapping effect within the bands of Gallium Sulfide $(G a S)$. And also we model injection level dependences of free carriers trap in both conduction and valence bands at different localized state energies of Gallium Sulfide $(G a S)$.

\section{Trapping Effects of Shockley-Read- Hall recombination Mechanism}

The total density of the trap levels occupied by electrons, $n_{T}$ and holes, $p_{T}$, must equal the total density of traps $N_{T}$ [13]

$$
N_{T}=n_{T}+p_{T}
$$

The distribute on probability $f_{n}$ for the electron occupation of a trap located at energy $E_{T}$ in the band gap is derived using Fermi-Dirac statistics:

$$
f_{n}(T)=\frac{1}{1+\exp \left(\frac{E_{T}-E_{F}}{K_{B} T}\right)}
$$

The thermal equilibrium electron occupancy $\left(n_{0 T}\right)$ and hole occupancy $\left(p_{0 T}\right)$ of the localized state are given by [14]:

$$
n_{0 T}=\frac{N_{T}}{1+\exp \left(\frac{E_{T}-E_{F}}{K_{B} T}\right)}, \quad p_{0 T}=N_{T}-n_{0 T}
$$

The probability of electron capture, $U_{c n}$ and $U_{e n}$ emission of holes $U_{e p}$ are:

$$
U_{c n}=C_{n T} n_{0} N_{T}\left(1-f_{n}\right), \quad U_{e n}=e_{n} N_{T} f_{n}
$$

The probability of hole capture $\left(U_{c p}\right)$, and emission of holes $U_{e p}$ are:

$$
U_{c p}=C_{p T} p_{0} N_{T} f_{n}, \quad U_{e p}=e_{p} N_{T}\left(1-f_{n}\right)
$$

By using principle of detailed balance, hole emission rate $e_{p}$ and electron emission rate $e_{n}$ are [15]:

$$
e_{p}=\frac{C_{p T} p_{0} f_{n}}{\left(1-f_{n}\right)}, \quad e_{n}=\frac{C_{n T} n_{0}\left(1-f_{n}\right)}{f_{n}}
$$

The density of electrons $\left(n_{0}\right)$ and the density of holes $\left(p_{0}\right)$ are:

$$
n_{0}=N_{c} \exp \left(-\frac{E_{c}-E_{F}}{K_{B} T}\right), \quad p_{0}=N_{v} \exp \left(-\frac{E_{F}-E_{v}}{K_{B} T}\right)
$$

The intrinsic energy level, $E_{i}$ is:

$$
n_{i}=N_{c} \exp \left(-\frac{E_{c}-E_{i}}{K_{B} T}\right), \quad p_{0}=N_{v} \exp \left(-\frac{E_{i}-E_{v}}{K_{B} T}\right)
$$

Then, electron emission rate and the hole emission rates are:

$$
e_{n}=C_{n T} n_{1}, \quad e_{p}=C_{p T} p_{1}
$$

where,

$$
n_{1}=n_{i} \exp \left(-\frac{E_{T}-E_{i}}{K_{B} T}\right) \text { and } p_{1}=n_{i} \exp \left(-\frac{E_{i}-E_{T}}{K_{B} T}\right)
$$

are the concentrations of electrons and holes in the conduction and valence bands. 
The photo-generated charge carrier neutrality equation for the system with an electron trap center and hole trap center gives [15]

$$
\delta p=\delta n+\delta n_{T} \quad \text { and } \quad \delta n=\delta p+\delta p_{T}
$$

In a system with dominant recombination through the impurity level, the rate of electron accumulation $d n / d t$ in the conduction band is given by:

$$
\frac{d n}{d t}=G-\left(U_{c n}-U_{e n}\right)
$$

Where, $G$ is the total generation rate, $U_{e n}$ is the rate of electron emission and $U_{c n}$ is the rate of electron capture by the localized state.

The rate of hole accumulation $(d p / d t)$ in the valence band is also given

$$
\frac{d p}{d t}=G-\left(U_{c p}-U_{e p}\right)
$$

The above two equation involves the accumulation of the total free charge carriers (thermal equilibrium and photo-generated).

The rates of accumulation of photo-generated charge carriers in the conduction and the valence bands were:

$\frac{\delta n}{d t}=G_{0}-\left(U_{c \delta n}-U_{e \delta n}\right) \quad$ and $\quad \frac{\delta p}{d t}=G_{0}-\left(U_{c \delta p}-U_{e \delta p}\right)$

where $G_{0}$ is the optical generation rate. The term $\left(U_{c \delta n}-\right.$ $\left.U_{e \delta n}\right)$ in Eqn. (14) involves the sum of the SRH recombination rate with trapping effect $\left(U_{S H R}^{\prime}\right)$ and the rate of accumulation photo-generated electrons $\left(d \delta n_{T} / d t\right)$ holes $U_{c \delta p}-U_{e \delta p}$ at the localized center given by [16]:

$$
\begin{gathered}
U_{c \delta n}-U_{e \delta n}=C_{n T}\left[p_{0 T} \delta n-\left(n_{o}+\delta n+n_{1}\right) \delta n_{T}\right]= \\
\left(U_{S H R}^{\prime}+\frac{d \delta n_{T}}{d t}\right. \\
U_{c \delta p}-U_{e \delta p}=C_{p T}\left[n_{0 T} \delta p+\left(p_{o}+\delta p+p_{1}\right) \delta n_{T}\right]=U_{S H R}^{\prime}
\end{gathered}
$$

The accumulation of free carriers in the respective conduction and valence bands are given by:

$$
\begin{array}{r}
\frac{d \delta n}{d t}=G_{0}-C_{n T}\left[p_{0 T} \delta n-\left(n_{o}+\delta n+n_{1}\right) \delta n_{T}\right]= \\
G_{0}-\left(U_{S H R}^{\prime}+\frac{d \delta n_{T}}{d t}\right.
\end{array}
$$

$\frac{d \delta p}{d t}=G_{0}-C_{p T}\left[n_{0 T} \delta p+\left(p_{o}+\delta p+p_{1}\right) \delta n_{T}\right]=G_{0}-\left(U_{S H R}^{\prime}\right)$

The rate of accumulation of photo-generated electrons at the localized centre can be:

$$
\begin{array}{r}
\frac{d \delta_{n T}}{d t}=C_{n T}\left[p_{0 T} \delta n-\left(n_{o}+\delta n+n_{1}\right) \delta n_{T}\right]-C_{p T}\left[n_{0 T} \delta p\right. \\
\left.+\left(p_{o}+\delta p+p_{1}\right) \delta n_{T}\right]
\end{array}
$$

Applying the steady-state condition to Eqn.19,

$$
\tau_{p 0} \delta n_{T}^{2}+b \delta n_{T}-c=0
$$

where,

$$
\begin{gathered}
\tau_{n 0}=\frac{1}{C_{n T} N_{T}} \text { and } \tau_{p 0}=\frac{1}{C_{p T} N_{T}}, \\
b=\tau_{n 0}\left(p_{o}+\delta p+p_{1}\right)+\tau_{p 0}\left(n_{o}+\delta n+n_{1}+p_{o T}\right), \\
c=\left(\tau_{p 0} p_{o T}-\tau_{n 0} n_{o T}\right) \delta_{p}
\end{gathered}
$$

The lifetime $\tau_{n 0}$ is often called the minimum lifetime for electrons when all the localized states are empty, and $\tau_{p 0}$ is the minimum lifetime for holes when all localized states are occupied by electrons.

The density of the excess electrons trapped at the localized center are:

$$
\delta n_{T}=\frac{\left(\tau_{p 0} p_{0 T}-\tau_{n 0} n_{0 T}\right) \delta p}{\tau_{n 0}\left(p_{0}+p_{1}+\delta p\right)+\tau_{p 0}\left(n_{0}+n_{1}+p_{0 T}+\delta p\right)}
$$

The fraction of photo-generated electrons and holes trapped are:

$$
I_{n}=\frac{\delta n_{T}}{\delta n}=\frac{\tau_{p 0} p_{0 T}-\tau_{n 0} n_{0 T}}{\tau_{n 0}\left(p_{0}+p_{1}+n_{0 T}+\delta N\right)+\tau_{p 0}\left(n_{0}+n_{1}+\delta N\right)}
$$

$$
I_{p}=\frac{\delta p_{T}}{\delta p}=\frac{\tau_{n 0} n_{0 T}-\tau_{p 0} p_{0 T}}{\tau_{n 0}\left(p_{0}+p_{1}+\delta P\right)+\tau_{p 0}\left(n_{0}+n_{1}+p_{0 T}+\delta P\right)}
$$

where $\delta N$ is the total excess electron concentration generated in the conduction band and $\delta P$ is the total excess hole concentration generated in the valence band. The subscripts $n$ and $p$ represent the electrons and holes concentration.

The fraction of photo-generated hole in the valence band and electrons in conduction bands are:

$$
I_{P}=\frac{\delta p}{\delta P}=\frac{\tau_{n 0}\left(p_{0}+p_{1}+\delta P\right)+\tau_{p 0}\left(n_{0}+n_{1}+p_{0 T}+\delta P\right)}{\tau_{n 0}\left(p_{0}+p_{1}+n_{0 T}+\delta P\right)+\tau_{p 0}\left(n_{0}+n_{1}+\delta P\right)}
$$

$I_{N}=\frac{\delta n}{\delta N}=\frac{\tau_{n 0}\left(p_{0}+p_{1}+n_{0 T}+\delta N\right)+\tau_{p 0}\left(n_{0}+n_{1}+\delta N\right)}{\tau_{n 0}\left(p_{0}+p_{1}+n_{0 T}+\delta P\right)+\tau_{p 0}\left(n_{0}+n_{1}+\delta P\right)}$

\section{Numerical Result and Discussion}




\subsection{Variation of Free Carries Trap With Tem- perature}

Figure 2: shows that the ratio of the concentration of trapped carriers to the corresponding concentration of free carriers, (a) at high temperature $(700 \mathrm{~K})$, (b) at different temperatures and (c) at different impurity density of trap state. We used doping concentration of $N_{a}=1 \times 10^{17} \mathrm{~cm}^{-3}$, illumination of $G_{0}=$ $1 \times 10^{25} \mathrm{~cm}^{-3} \mathrm{~s}^{-1}$ and $E_{a}=0.01 \mathrm{eV}$.

Figure 2 (a) shows different regions of localized states with the conduction or valence bands at high temperature and the energy level dependence of trap divided into five regions based on the interactions of the localized states with the conduction or valence bands.

Figure 2 (b) shows the effects of free carriers trap at different temperatures. As we can see from the figure, at low temperatures, the carriers have insufficient thermal energy to become excited into the conduction (or valence) band, reducing the density of ionized impurities. Free carrier traps have more effects at low temperatures when compared to high temperatures. Only deep levels can trap free carriers at higher temperatures. The minority carrier traps farther away above the intrinsic level becomes more effective with decreasing temperature. The probability of emission of carriers by the Centre increases with increasing temperature. The localized Center closer to the intrinsic level become the most dominant electron trap Centre at lower temperatures and localized Centre farther away above the intrinsic level become the most dominant electron trap Centre at lower temperatures.

Figure 2 (c) shows the effects of free carriers trap at different impurity density of states at room temperature. As the total impurity density of trap states increases, the thermal equilibrium concentration of holes and electrons at the trap level increases. As impurity density of trap states increases, the majority carrier traps in the acceptor level and the minority carrier traps in the donor level increases. The minority carrier traps farther away above the intrinsic level become more effective with increasing impurity density of traps. The intensity of trapping increases with increasing impurity density of traps.

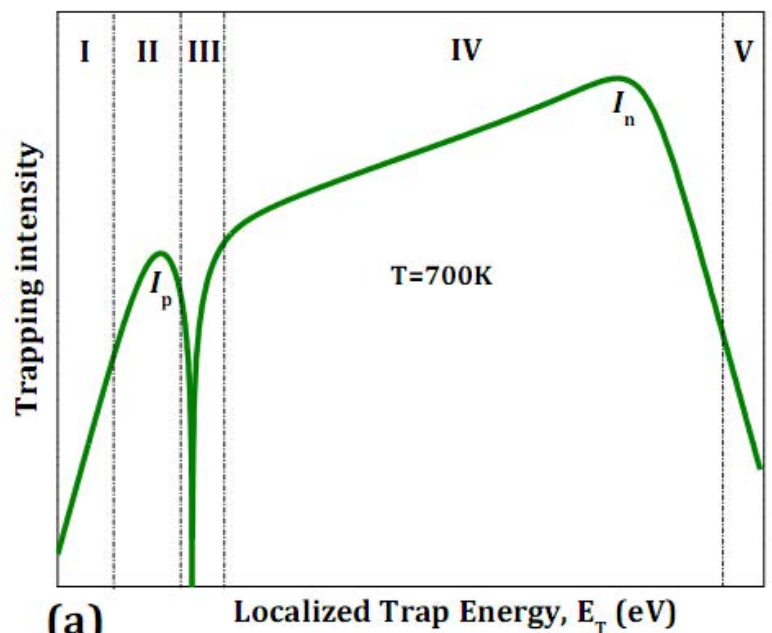

The energy level dependence of trap can be divided into five regions based on the interactions of the localized states with
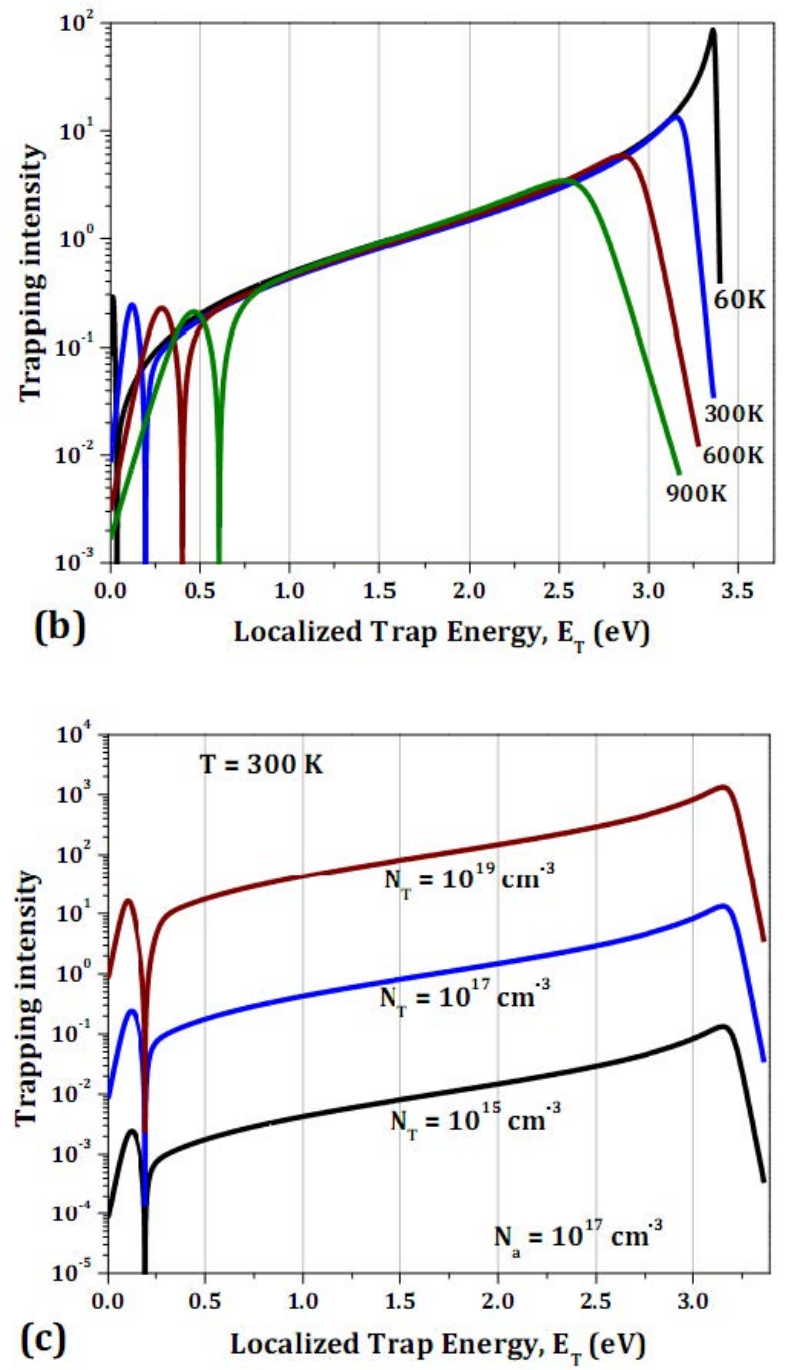

Figure 2. The ratio of the concentration of trapped carriers to the corresponding concentration of free carriers as a function of localized state energy for Gallium Sulfide $(\mathrm{GaS})$ compound semiconductor sample

the conduction or valence bands:

I. Acceptor region: This region comprises all the energy levels very close to the valence band edge. Most of the impurities are in the $n_{T}$ states and the holes emission rate is very high (concentration of holes in the valence band is greater than thermal equilibrium concentrations of holes). Hence, the effects of the traps of a localized Centre becomes small and the localized Centre hence act as acceptor levels.

II. Holes trap region: This region encompasses far above the valence band edge and far below the Fermi level. Most localized states are still in the $n_{T}$ states and the hole emission rate has decreased (concentration of holes in the valence band is less than thermal equilibrium concentrations of holes). Thus, the hole trapping effect becomes dominant.

III. Recombination region: This region also encompasses all the regions very closer to the Fermi level, where the localized levels are partially ionized, the electron and holes capture rates are almost equal. So that the effects of free charge carriers traps are negligibly small and recombination becomes dominant in this region. 
IV. Electron trap region: This region comprises all the regions that are very close to the intrinsic Fermi level and most of the regions far above the intrinsic Fermi level. Most localized states are in the $p_{T}$ states and the holes capture rate is very low. The electron trap effect becomes the most dominant.

V. Donor region: This region consists of all the localized centres closer to the conduction band. All the localized states in this region are in the $p_{T}$ states. Hole capture rate is negligibly small and the electron emission rate is extremely very high (the concentration of electrons in the conduction band greater than thermal equilibrium concentration of holes and total density of traps). Hence, the free carriers trap effect in this region is very negligible and all the localized centres act as donor levels.

\subsection{Injection Level Dependence of Free Carri- ers Trap}

Figure 3 shows the injection level dependence of (a) the ratio of trapped excess carriers to conduction excess carriers $\left(I_{n b}\right)$ for different localized energy levels and (b) the densities of excess holes in the valence band $(\delta p)$, excess electrons in the conduction band $(\delta n)$ and trapped excess electrons $\left(\delta n_{T}\right)$ in a localized state of energy $0.80 \mathrm{eV}$ where maximum trapping takes place at room temperature. The results represent Gallium Sulfide with a carrier density $1 \times 10^{18} \mathrm{~cm}^{-3}$ and a trap density $1 \times 10^{16} \mathrm{~cm}^{-3}$ at room temperatures. As one can see from Figure 3 (a) the room temperature, low-level injection trapping effect for excess electrons is very low at lower and higher localized energy levels and reach a maximum value at a localized state closer to $0.80 \mathrm{eV}$. However, this ratio for all the centers diminishes to zero at a high injection level as the density of excess electrons in the conduction band exceeds the density of the impurity state in the localized center.

The low injection level density of excess electrons trapped in the localized state $\left(\delta n_{T}\right)$ can exceed the corresponding density of excess electrons in the conduction band $(\delta n)$ as shown in Figure 3(b). The variations of $\delta p, \delta n$ and $\delta n_{T}$ with $G_{0}$ is also the same at very low injection regimes. However, as the injection level increases all the localized states are filled with excess electrons $\left(\delta n_{T} \approx N_{T}\right)$ and hence $\delta n_{T}$ remained constant and $\delta n$ increases abruptly until it becomes equal to $\delta p$ at higher injection level (as seen in figure 3(b) below.

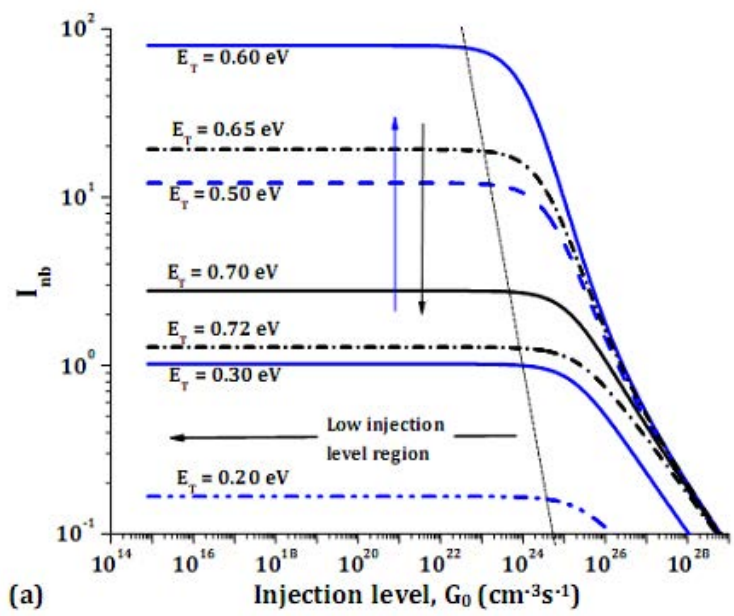

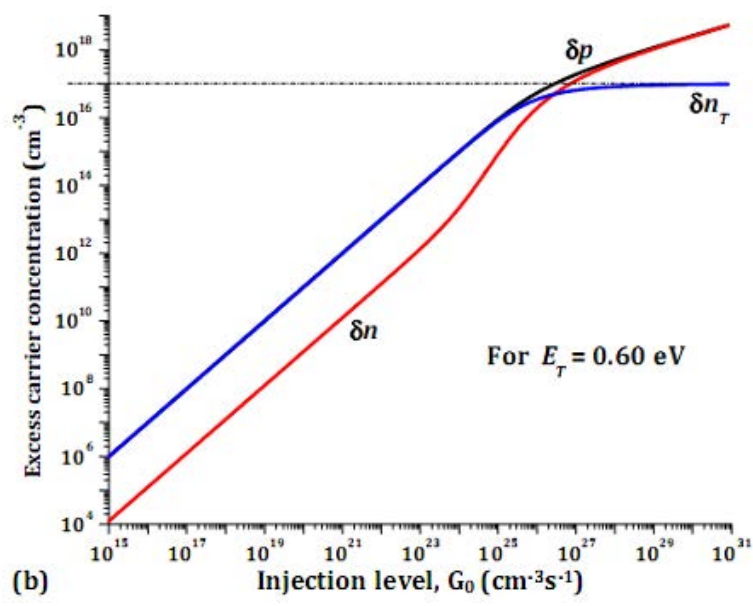

Figure 3. Injection level dependence of (a) the ratio of trapped excess carriers to conduction excess carriers for different localized energy levels and (b) the densities of excess holes in the valence band $(\delta p)$, excess electrons in the conduction band $(\delta n)$ and trapped excess electrons $\left(\delta n_{T}\right)$ in a localized state of energy $0.80 \mathrm{eV}$ where maximum trapping is occurred at room temperature for Gallium Sulfide $G a S$ sample.

\section{Summary and Conclusion}

In the present work, we have studied the effects of temperature on the free carriers trap of Gallium Sulfide $(G a S)$ in Shockley read hall recombination mechanism. To do this, we have seen different trap level energies between conduction and valence bands. In Shockley-Read-Hall (SRH) recombination, the recombination of EHPs may take place at localized traps with states in the band gap of the material. This process involves the capture of electrons from the conduction band (or holes from the valence band) by the trap, followed by the recombination with holes in the valence band (or electrons in the conduction band). When occupied by an electron, the trap level is in the $n_{T}$ state, and when occupied by a hole it is in the $p_{T}$ state. After the electron has been emitted, the center finds itself in the $p_{T}$ state and subsequently emits a hole, returning to the $n_{T}$ state, and then the cycle repeats. Just like a donor level, if the $n_{T}$ state is neutral, then pT is positively charged, and vice versa.

We have classified localized state energy into five regions at high-temperature regime $(700 K)$. The position of Fermi-level plays a crucial role in determining these regions. Accordingly, the energy level very close to the valence band edge is the acceptor region, where, the energy level very close to the conduction band is donor regions. Holes trap region is the region above the valence band edge and far below the Fermi level and electron trap region is very close to the intrinsic Fermi level and most of the regions far above the intrinsic Fermi level. For all different impurity density of states at room temperature $(300 K)$, at low localized trap energy, there are holes trapping effects and for a wide range of localized trap energy electron trapping effects. 


\section{REFERENCES}

[1] Lieth, R. M. A. Preparation and Crystal Growth of Materials with Layered Structures; Reidel, D., Ed.; Reidel: Dordrecht, Holland, 1977.

[2] G. Akhundov, I. G. Aksyanov, and G. M. Gasumov, Sov. Phys. Semicond. 3, 767 1969!.

[3] . M. I. Karaman and V. P. Mushinskii, Sov. Phys. Semicond. 4, 662 1970!.

[4] . A. Cingolani, A. Minafra, P. Tantalo, and C. Paorici, Phys. Status Solidi A 4, K83 1971!.

[5] T. Aono, K. Kase, and A. Kinoshita, J. Appl. Phys. 74, 28181993 !.

[6] . A. Mercier, E. Mooser, and J. P. Voitchovsky, J. Lumin. 7, 2411973 !.

[7] . A. Mercier and J. P. Voitchovsky, J. Phys. Chem. Solids 36, 14111975 !.

[8] . C.W. Tang and S.A. VanSlyke, Appl. Phys. Lett. 51, 913 (1987).

[9] . J.H. Burroughes, D.D.C. Bradley, A.R. Brown, R.N. Marks, K. Mackey, R.H. Friend, P.L. Burn, and A.B. Holmes, Nature (London) 347, 539 (1990).

[10] . W. Shockley and W.T. Read, Phys. Rev. 87, 835 (1952).

[11] . R. N. Hall, Electron-hole recombination in Germanium, Phys. Rev., 87 (1952), p. 387.

[12] . R. N. Hall, Electron-hole recombination in Germanium, Phys. Rev., 87 (1952), p. 387.

[13] . W. Shockley and W. T. Read, Statistics of the recombinations of holes and electrons, Phys. Rev., 87 (1952), pp. 835-842.

[14] . Sheng S. Li, Semiconductor Physical Electronics, Second Edition, USA: Springer Science+Business Media, LLC, 233 Spring Street, New York, NY 10013, USA, 2006.

[15] . Varshni Y. P., ’Temperature dependence of the energy gap in semiconductors," Physica, vol. 34, p. 149, 1967.

[16] . A. Mitonneau, A. Mircea, G.M. Martin, D. Pons, ”Electron and hole capture cross-sections at deep centers in gallium arsenide," HAL, pp. 853-860, 1979. 